Supporting Information

\title{
POST-AdsoRption WoRK FUnCTION TUNING via Hydrogen Pressure Control
}

\author{
Hermann Edlbauer, Egbert Zojer, and Oliver T. Hofmann* \\ Institute for Solid State Physics, NAWI Graz, Graz University of Technology, 8010 Graz, \\ Austria
}

Tel.: $\quad+433168738465$

Fax.: $\quad+433168738466$

Mail: $\quad$ o.hofmann@tugraz.at 


\section{A. Supercell Geometries}

In this chapter we describe the particular supercell geometries that were investigated on $\mathrm{Cu}(111)$ and $\mathrm{Ag}(111)$. Due to the single bond of the OH-group of the TFBD molecule to its phenyl ring it is freely rotatable along the bonding direction. By rearranging the TFBQ and TFBD molecules on the adsorption sites with gradually increasing TFBD fraction, $f$, and different alignments of the rotatable $\mathrm{OH}$-groups, we found that for the ground state energy the alignment of the $\mathrm{OH}-$ bonds is crucial. To converge into the global energy minimum in the geometry optimization procedures it is thus necessary to rotate those $\mathrm{OH}$-groups in such a way that the hydrogen comes in the vicinity of an oxygen atom of a neighboring TFBQ molecule. This then leads to the formation of a hydrogen bond.

(a) Geometry 1

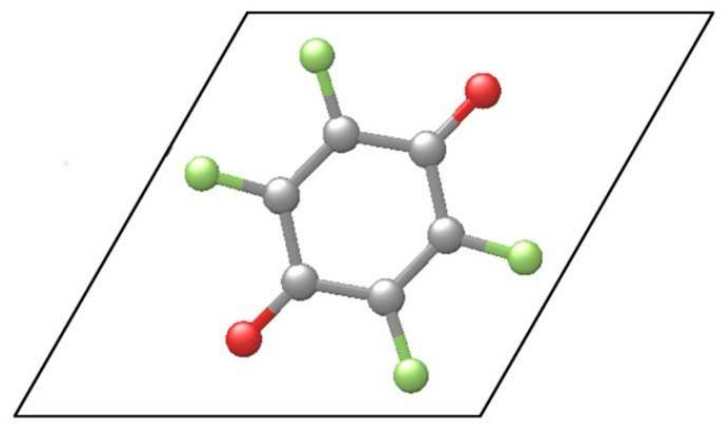

(c) Geometry 3

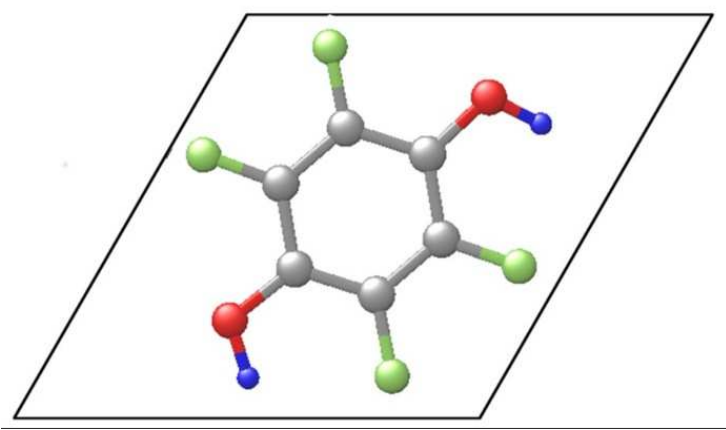

(b) Geometry 2

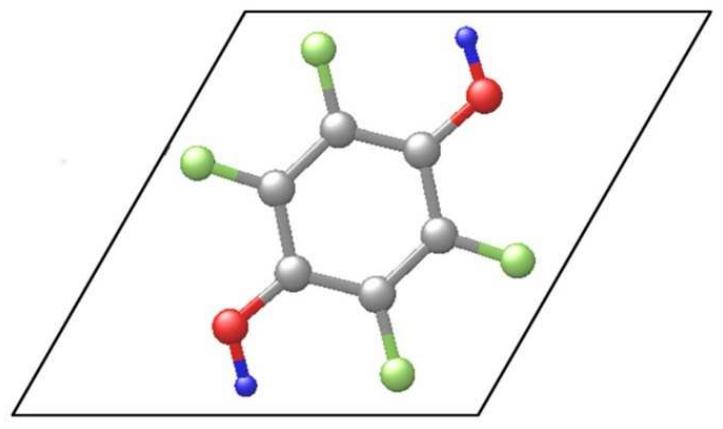

(d) Geometry 4

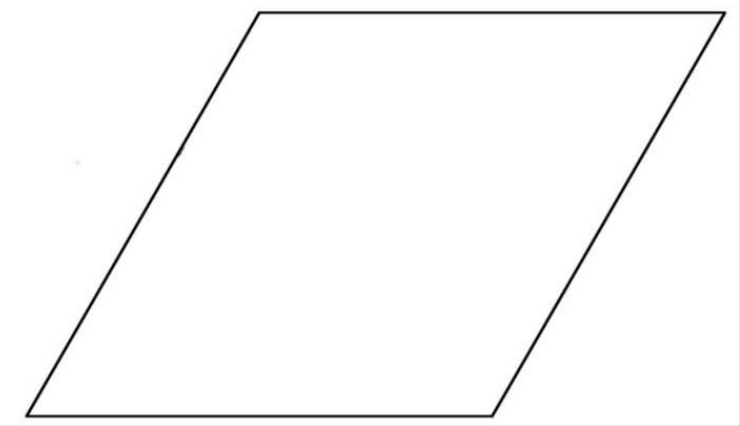

Figure S1. Geometries that were arranged on the adsorption sites of supercells containing TFBQ and TFBD. (a) Geometry 1 for TFBQ; (b) Geometry 2 for TFBD with neighboring TFBQ molecules; (b) Geometry 3 for TFBD with neighboring TFBD molecules. (c) Geometry 4 for empty adsorption site. 
It turns out that four geometries, as sketched in Figure S1 serve as basic building blocks for the supercell geometries. To identify a certain supercell geometry based on its building blocks we introduced the following nomenclature. Starting from the lower, left adsorption site of a supercell the respective sub-geometries shown in Figure $\mathrm{S} 1$ are labelled by the numbers 1, 2, 3 and 4. A new row is indicated by a slash symbol "/". The supercell geometry shown in Figure S1 of the main paper then for instance would be labelled as "21/12". By proper alignment of those building blocks a set of supercell geometries were created that served as starting points for geometry optimizations. A list of the supercell geometries that were investigated within this work is quoted in Table S1. The corresponding relaxed supercell geometries of mixed monolayers and sub-monolayers are shown for adsorption upon $\mathrm{Cu}(111)$ in Figure S2 and for $\mathrm{Ag}(111)$ in Figure S3.

Depending on the neighboring molecules above and below a unit cell for TFBD in the adsorption site two alignments of the $\mathrm{OH}$-bonds minimize the ground state energy: Geometry 2 is preferable if hydrogen bonds are formed to neighboring TFBQ molecules -- the $\mathrm{OH}-$ group is rotated such that the hydrogen approaches the oxygen atom of the neighboring TFBQ molecule as close as possible. For neighboring TFBD molecules geometry 3 minimizes the ground state energy - on $\mathrm{Cu}(111)$ for instance $E_{33 / 33}-E_{22 / 22}=-0.63 \mathrm{eV}$. (See also discussion in the main text)

In mixed layers of electron-donating and -accepting molecules or SAMs cases of superstructure formation and phase separation were reported. ${ }^{1,2}$ To check if phase separation is likely to occur for the investigated system, a set of supercell geometries with different arrangements of TFBQ and TFBD molecules at $50 \%$ TFBD fraction were evaluated. We found that checkerboard motifs are always more stable than structures where molecules are arranged in rows or clusters. However the difference in the total energies is just a few meV: on $\mathrm{Cu}(111)$ for instance $E_{12 / 21}-E_{12 / 12}=-4.5 \mathrm{meV}$ and $E_{12 / 21}-E_{11 / 22}=-1.5 \mathrm{meV}$. This shows that alternating patterns are energetically favorable, and hence, for a mixed monolayer of TFBQ and TFBD phase separation is not expected. 
Table S1. Supercell geometries for the simulation of mixed monolayers and submonolayers.

\begin{tabular}{lccc}
\hline \multicolumn{1}{c}{ Type of Adsorbate } & \multicolumn{2}{c}{ Supercell Geometries } \\
\hline mixed monolayers & $11 / 21$, & $12 / 21$ & and $13 / 23$ \\
sub-monolayers of TFBQ & $11 / 41$, & $14 / 41$ & and $14 / 44$ \\
sub-monolayers of TFBD & $44 / 24$, & $42 / 24$ & and 43/23 \\
limiting cases & $11 / 11$, & $33 / 33$ & and 44/44 \\
\hline
\end{tabular}

(a) $14 / 44$

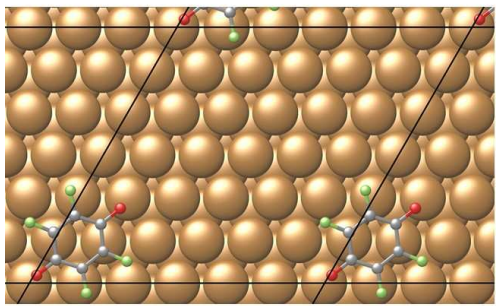

(d) $24 / 44$

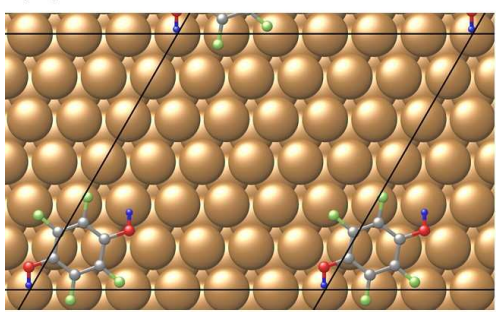

(g) $12 / 11$

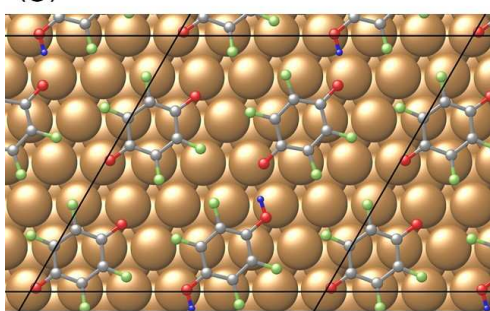

(b) $14 / 41$

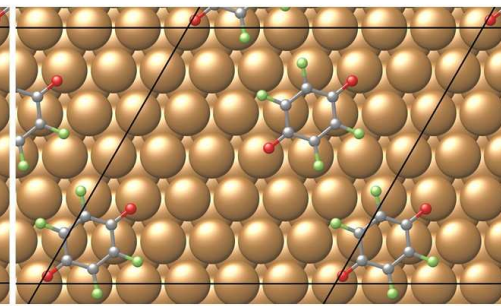

(e) $24 / 42$

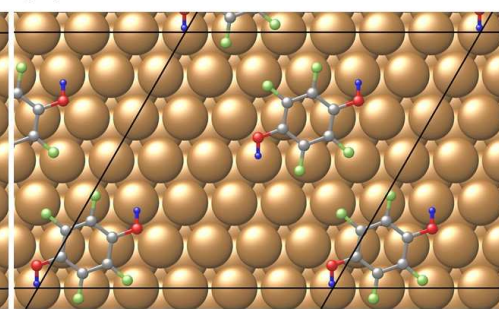

(h) $21 / 12$

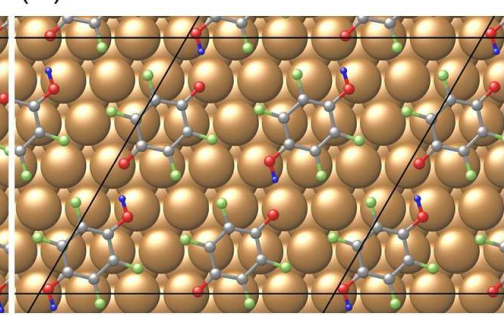

(c) $14 / 11$

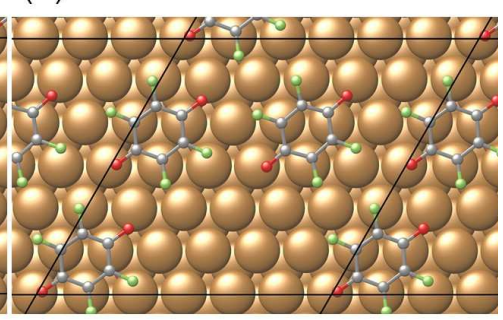

(f) $34 / 32$

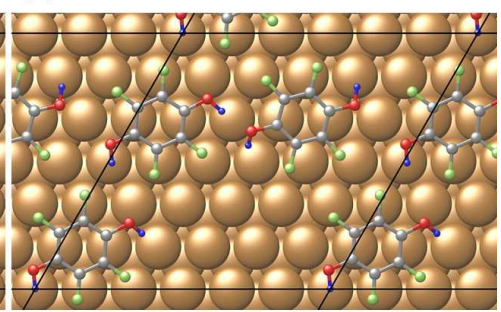

(i) $13 / 23$

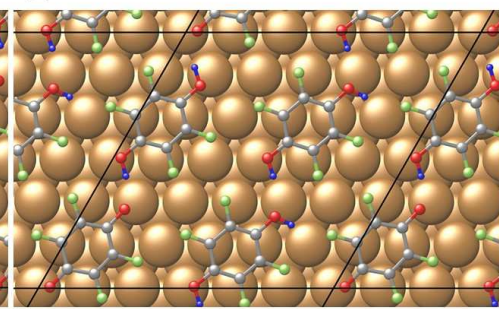

Figure S2. Top views on supercell geometries of submonolayers of (a-c) TFBQ and (d-f) TFBD and (g-i) mixed monolayers on $\mathrm{Cu}(111)$. 
(a) $14 / 44$

(b) $14 / 41$

(c) $14 / 11$

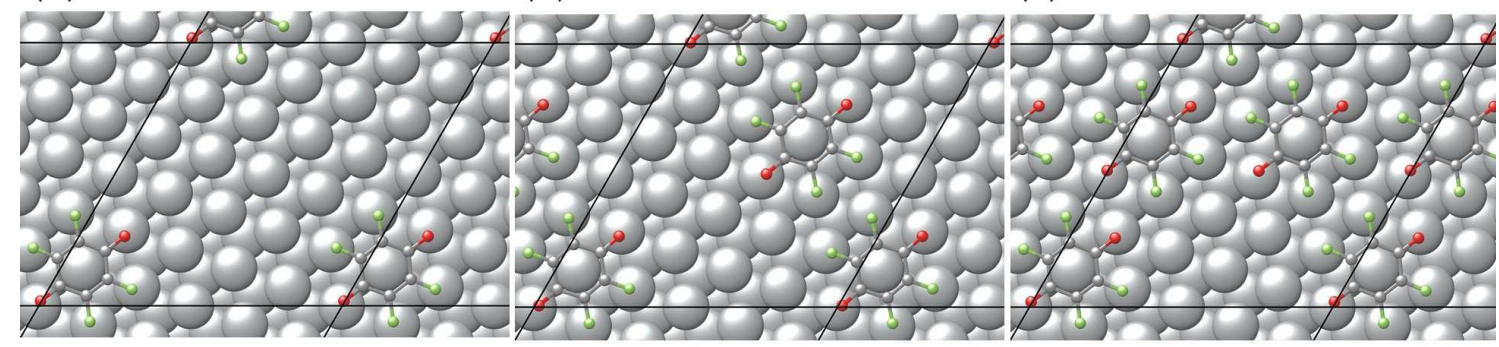

(d) $42 / 44$

(e) $42 / 24$

(f) $43 / 23$

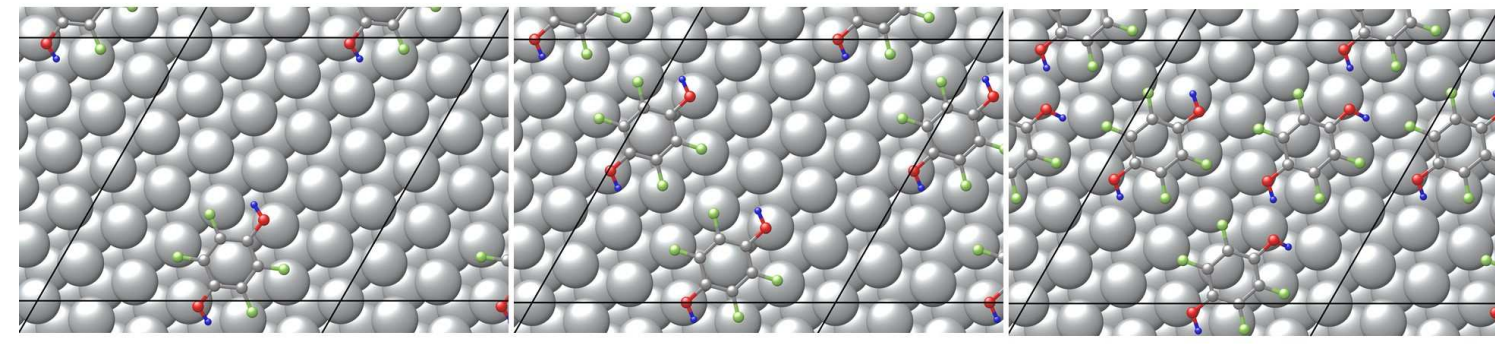

(g) $12 / 11$

(h) $12 / 21$

(i) $13 / 23$

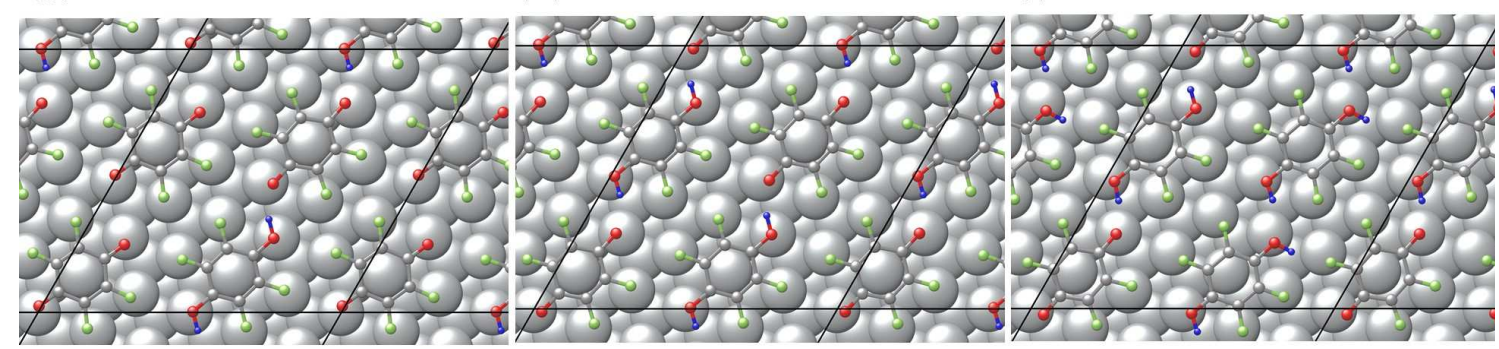

Figure S3. Top views on supercell geometries of submonolayers of (a-c) TFBQ and (d-f)

TFBD and (g-i) mixed monolayers on $\operatorname{Ag}(111)$. 
(a) $11 / 11$ on $\mathrm{Cu}(111)$

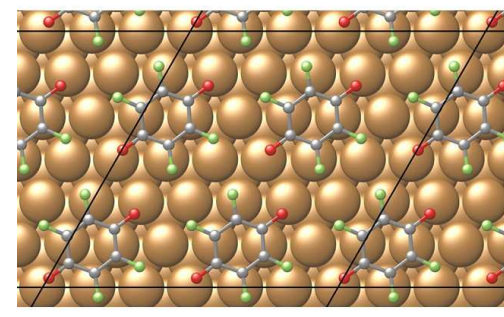

(d) 24/44 on $\mathrm{Ag}(111)$

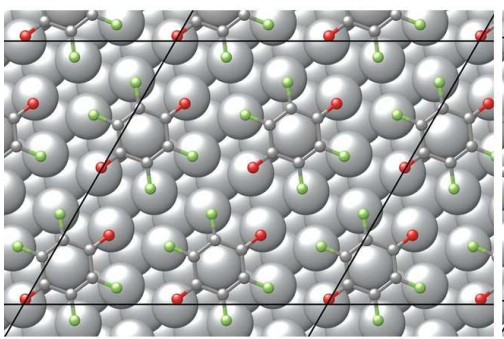

(b) $33 / 33$ on $\mathrm{Cu}(111)$

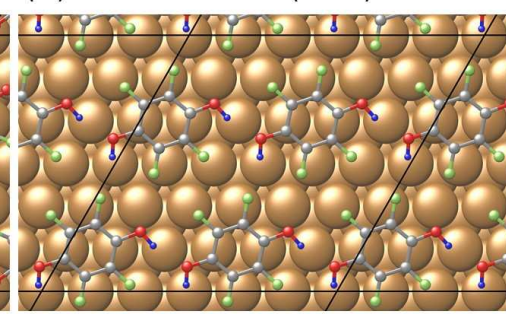

(e) $33 / 33$ on $\mathrm{Ag}(111)$ (c) $44 / 44$ on $\mathrm{Cu}(111)$

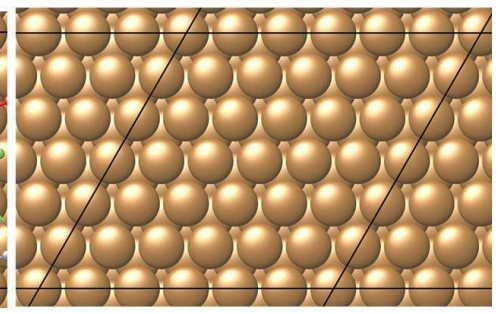

(f) $44 / 44$ on $\mathrm{Ag}(111)$

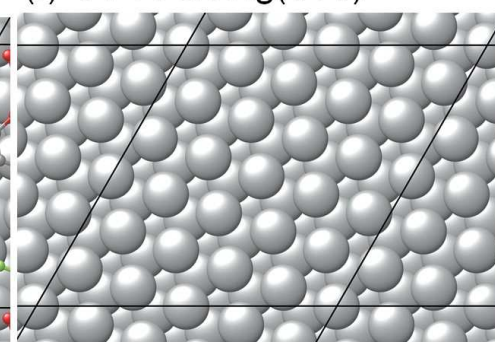

Figure S4. Top views on supercell geometries of submonolayers for the limiting cases of homogeneous monolayers fully covered with TFBQ or TFBD and a clean (111)-surface for (a-b) $\mathrm{Cu}(111)$ and (d-f) $\operatorname{Ag}(111)$. 


\section{B. Sub-Monolayers of TFBD on Ag(111)}

The contributions of the molecular and bond dipole to $\Delta \Phi$ for sub-monolayers of TFBD on $\operatorname{Ag}(111)$ are displayed in Figure S5. Analyzing the evolutions one finds that the deviations from linearity mainly stem from the evolution of the molecular dipole, whereas the contributions from the bond dipole nearly follows a linear evolution (in fact, slightly parabolic coverage dependency) of the bond dipole.

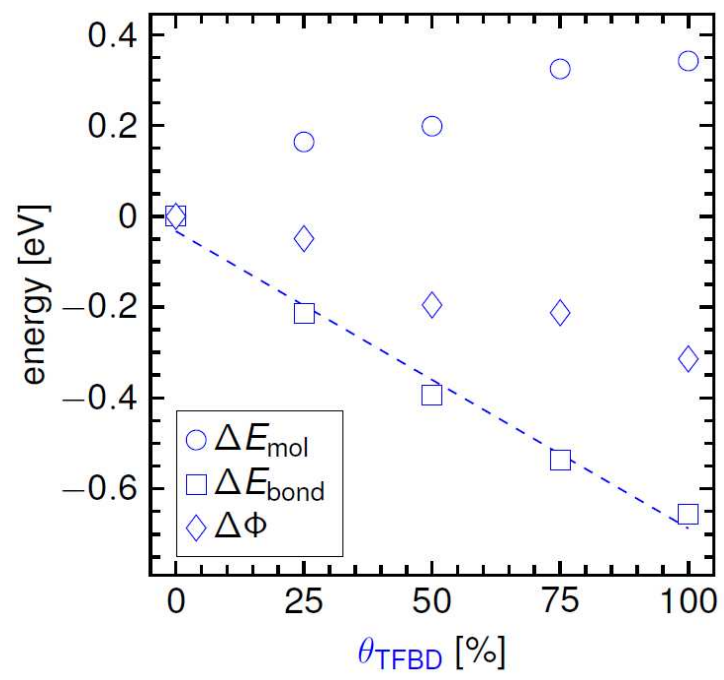

Figure S5. Evolutions of the contributions of the molecular dipole, $\Delta \mathrm{E}_{\mathrm{mol}}$, and the bond dipole, $\Delta \mathrm{E}_{\text {bond, }}$, to the work function modification, $\Delta \Phi$, for sub-monolayers of TFBD on $\operatorname{Ag}(111)$. 


\section{Adsorption Heights and Charges in Mixed Monolayers}

For $\mathrm{Cu}(111)$ and $\mathrm{Ag}(111)$ the adsorption heights are shown in S6a and Figure S6b. Comparing the adsorption heights for TFBD in mixed monolayers (blue filled diamonds) to sub-monolayers (blue diamonds) one finds that for both coinage metals TFBD comes closer to the surface the larger the number of neighboring TFBQ molecules is. The decrease of the adsorption height in mixed monolayers likely stems from the electrostatic attraction with TFBQ. Conversely the TFBQ molecules increase their adsorption heights only on $\mathrm{Cu}(111)$. On $\operatorname{Ag}(111)$, where the adsorption height of a homogeneous TFBQ monolayer is about $0.4 \AA$ larger than on $\mathrm{Cu}(111)$, the mean adsorption height stays approximately constant independent of $f$, which is also the case for the corresponding sub-monolayer case. Such an "equalization of the adsorption heights" was also observed experimentally for different mixed-monolayers of electron-accepting and -donating species. ${ }^{3}$ A further effect that was mentioned there was the modification of the electron spill out due to the adsorption of the molecules that affects neighboring adsorption sites. However, for the system investigated here, this is a minor effect.

(a) $\mathrm{Cu}(111)$

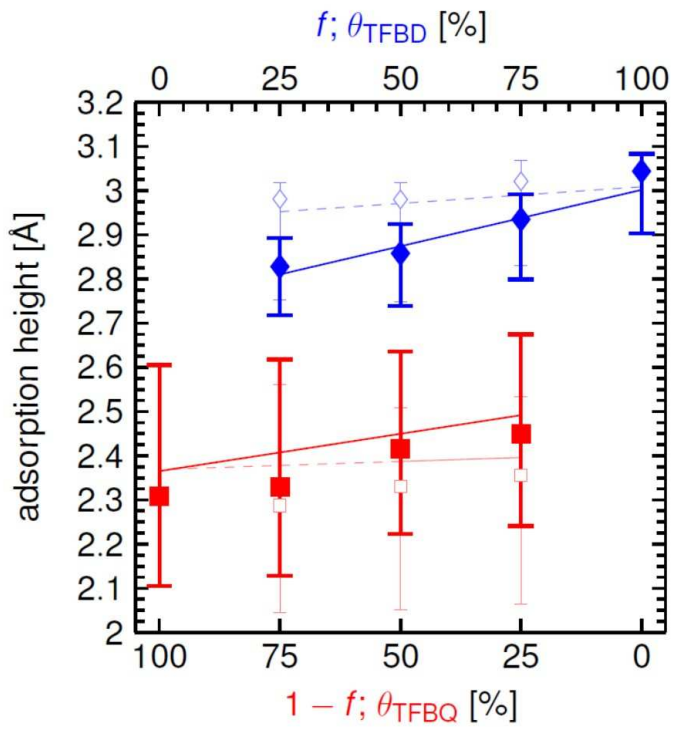

(b) $\mathrm{Ag}(111)$

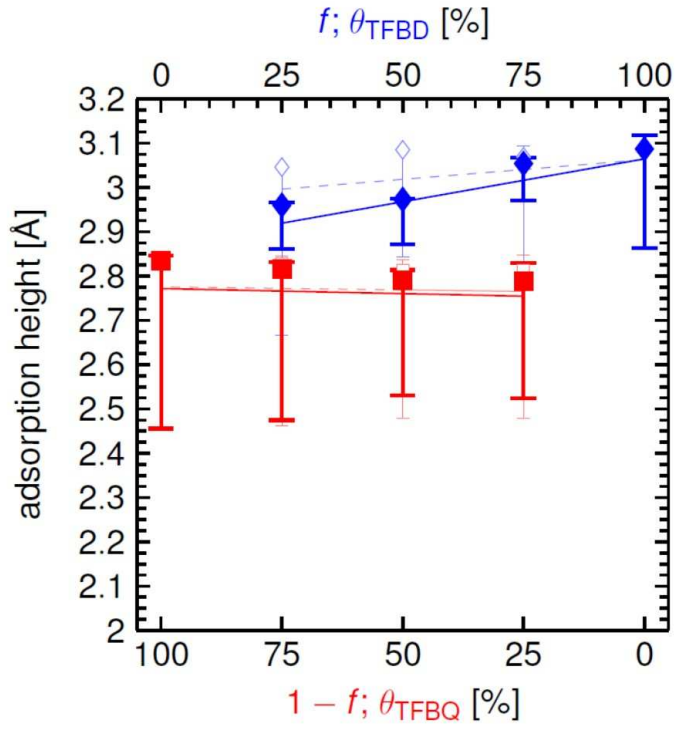

Figure S6. Adsorption heights of the atomic species of TFBQ (red squares) and TFBD (blue diamonds) in mixed monolayers on (a) $\mathrm{Cu}(111)$ and (b) $\mathrm{Ag}(111)$. The data points indicate the mean distance of the carbon backbone to the mean position of the upper metal layer. The upper error bar indicates the mean position of the fluorine atoms. The lower error bar 
describes the mean position of the oxygen atoms for TFBQ and of the hydrogen atoms for TFBD. The plotted lines indicate linear fits to the mean position of the TFBQ and TFBD molecules. For comparison further the results of the corresponding sub-monolayers are drawn - see smaller, half transparent, whitely filled symbols, with narrower "error" bars and dashed regression lines.

The Mulliken charges ${ }^{4}$ of the molecules of mixed monolayers upon adsorption on $\mathrm{Cu}(111)$ and $\operatorname{Ag}(111)$ - see Figure S7a and Figure S7b - strongly increase in magnitude for both TFBQ and TFBD upon intermixing. The larger the number of neighboring TFBQ (TFBD), the more the TFBD (TFBQ) molecules is charged.

(a) $\mathrm{Cu}(111)$

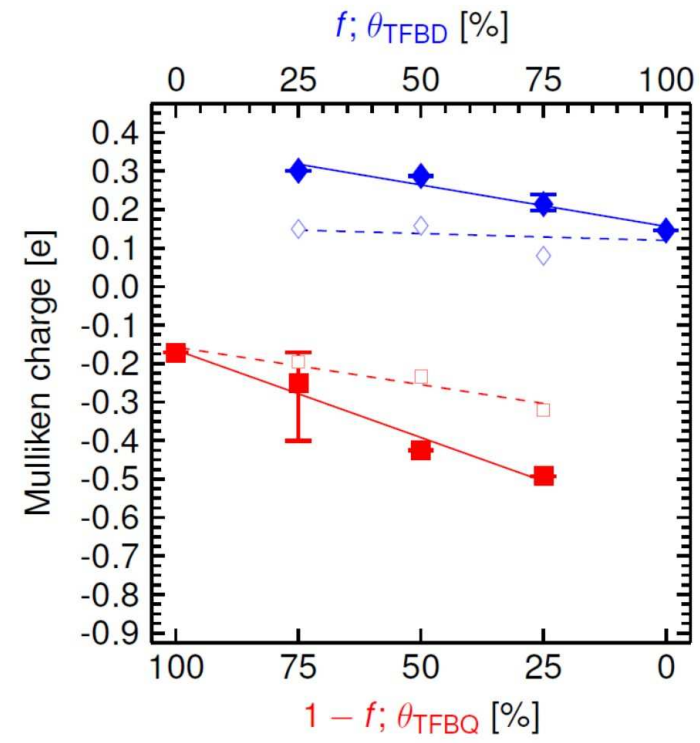

(b) $\mathrm{Ag}(111)$

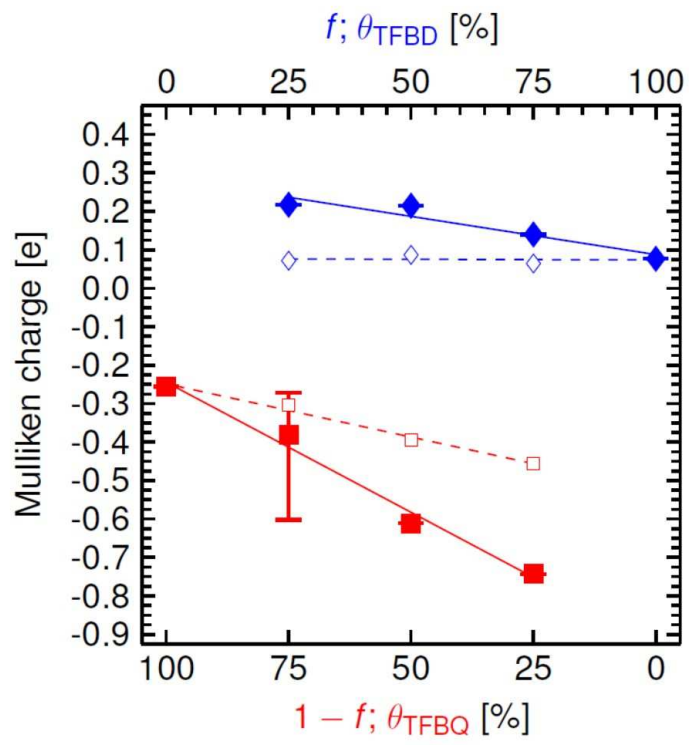

Figure S7. Mean values of the molecular charges of TFBQ (red squares) and TFBD (blue diamonds) in mixed monolayers on (a) $\mathrm{Cu}(111)$ and (b) $\mathrm{Ag}(111)$ calculated via the Mulliken partitioning scheme. The maximum and minimum values in mixed monolayers are shown via error-bars. To highlight the evolutions of the respective data sets moreover linear fits are plotted as lines. For comparison further the mean values of sub-monolayers at corresponding coverage are shown - see smaller, half transparent, whitely filled symbols, with narrower "error" bars and dashed regression lines.

We remark that in hypothetical, free-standing mixed monolayers charge-transfer from TFBD to TFBQ occurs, and that the TFBD-HOMO is found to be in resonance with the TFBQLUMO. Since there is no appreciable overlap of the wave functions in the coplanar monolayer, 
we speculate that this charge-transfer might be a spurious artefact of the PBE functional, akin to the situation discussed for other donor/acceptor pairs. ${ }^{5,6}$ Interestingly, the HOMO/LUMO resonance situation is lifted when the mixed layers are adsorbed onto the surface. This yields a situation that is in better accordance with chemical intuition and supports our earlier claim that $\mathrm{PBE}+\mathrm{vdW}{ }^{\text {surf }}$ yields reasonable electronic structures for metal $/$ molecule interfaces. ${ }^{7}$

\section{A different viewpoint on Fermi-level pinning in mixed layers}

The potential caused by the TFBD molecules explains the differences of the $\Delta \Phi(f)$-evolutions on $\mathrm{Cu}$ and $\mathrm{Ag}$. Due to the smaller adsorption height of TFBQ on $\mathrm{Cu}$ the distance to neighboring TFBD molecules is larger - compare situation 3 sketched in Figure S8a. The net charge formed at the TFBD-adsorption site due to the Pauli-pushback effect with the mirror charges in the metal lead to an electric field that weakly affects the energy of the TFBQLUMO due to its spatial position. For $\mathrm{Cu}$ therefore a smaller downshift of the LUMO (blue arrow pointing down in Figure S8b) and thus a smaller compensation from the bond dipole occurs (red arrow pointing up in Figure S8b). For mixed monolayers adsorbed upon Ag on the other hand the adsorption height of TFBQ is by about $0.45 \AA$ larger than on $\mathrm{Cu}$ - compare situation 2 sketched in Figure S8a. Therefore the electric field shifts the TFBQ-LUMO strongly down in energy (blue arrow pointing down in Figure S8b) what results in a strong compensation by the bond dipole (red arrow pointing up in Figure S8b). This further is reflected in the weaker decrease of $\Delta \Phi(f)$.

Note that within this idealized picture the LUMO-peak is modelled as a Delta-Distribution. However, due to the interaction of an adsorbed molecule with the adsorbent and neighboring molecules the molecular energy levels broaden. This causes that the Fermi-level-pinned LUMO-peak is not directly located at $E_{F}$ - as sketched in Figure S8. Further the LUMOenergy of the separated hypothetically, free standing monolayer in the Schottky-Mott-limit is not a constant for different conformations of the layer. Despite the higher work function of $\mathrm{Cu}$ $\left(\Phi_{\mathrm{Cu}}-\Phi_{\mathrm{Ag}} \approx 0.4 \mathrm{eV}\right)$ therefore it occurs that on $\mathrm{Ag}$ the Fermi-level-pinned LUMO-peak is closer to $E_{F}$. 
(a) Sketch of the different situation for the explanation of Fermi-level-pinning in mixed monolayers.

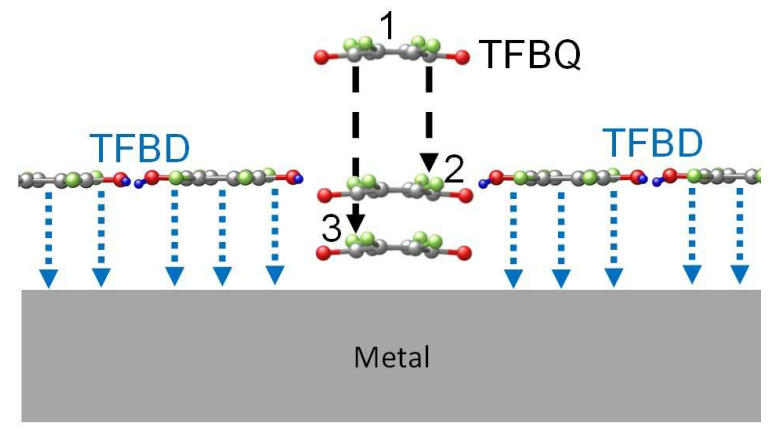

(b) Level alignment

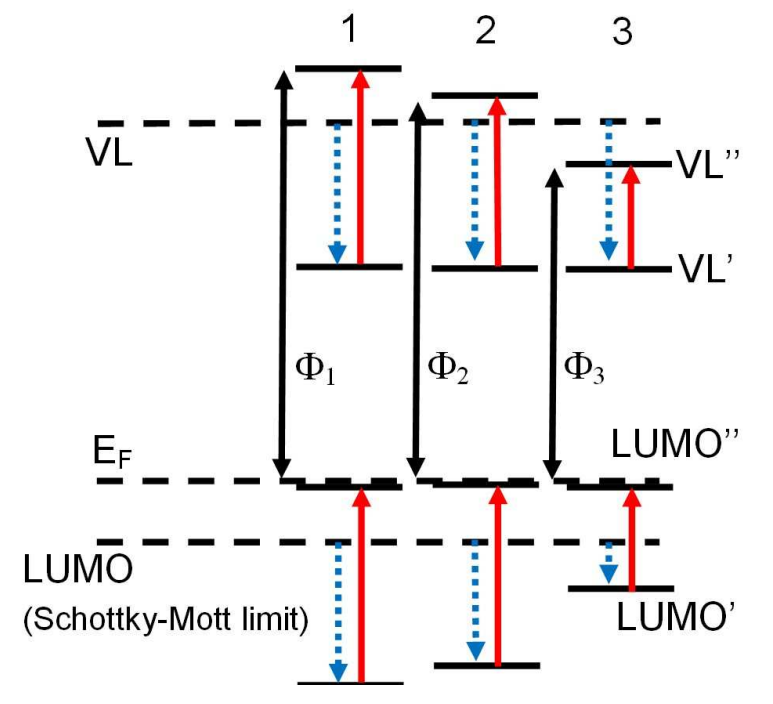

Figure S8. (a) Sketch for selected situations: (1) A TFBQ molecule outside a dipole field (blue dashed arrows) caused by a TFBD-sub-monolayer and a TFBQ molecule immersing in the dipole field with a (2) small and (3) large distance to the TFBD monolayer. Situations 2 and 3 resemble the situation of mixed monolayers on $\mathrm{Ag}$ and $\mathrm{Cu}$. (b) Energy level alignment due to Fermi level pinning: The TFBD-dipole field shifts the LUMO to LUMO` depending on the position of the TFBQ molecule. The shift of VL to $\mathrm{VL}^{\prime}$ on the other hand is independent of the TFBQ position. Due to Fermi-level-pinning charge rearrangements occur that shift the LUMO' and VL' to to LUMO“" and VL" ". The positions of TFBQ relative to the TFBD dipole field thus strongly affects $\Phi$. 


\section{E. Infra-red-spectra of TFBQ and TFBD}

To assess the quality of our calculations for the vibrational properties of the molecules, we calculated the the Infra-red (IR) spectra of TFBQ and TFBD in the gas phase (shown in figure S9). For comparison also the corresponding IR-spectra from the NIST database ${ }^{8}$ are given. The IR spectrum calculated via DFT shows overall good agreement with reference data from experiment. However, vibrations at intermediate wavenumbers $\left(<1200 \mathrm{~cm}^{-1}\right)$ are calculated at slightly lower energies than the peaks in the reference data set, while for very high wavenumbers $\left(>3000 \mathrm{~cm}^{-1}\right)$, the calculated energies are slightly too large. Interestingly, for the TFBD IR-spectrum a peak at $377 \mathrm{~cm}^{-1}$ is predicted by our calculations that does not appear in the reference data set.

(a) TFBQ

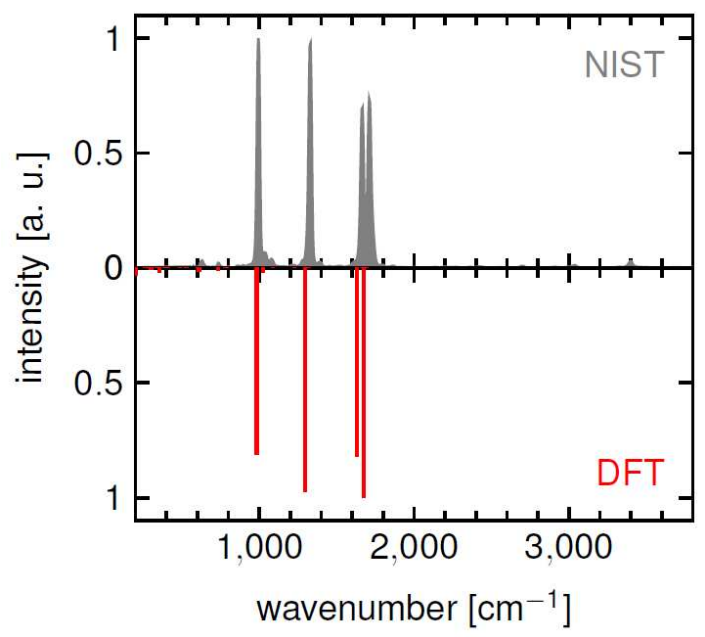

(b) TFBD

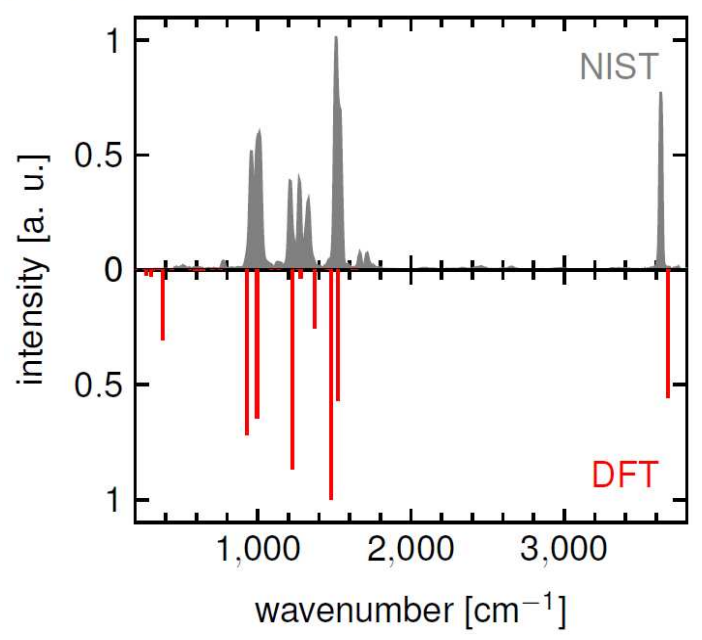

Figure S9. Comparison of IR-spectra of (a) TFBQ and (b) TFBD in gas from (top) NIST database ${ }^{8}$ to (bottom) DFT calculations.

\section{F. Extraction of Metal-Atoms due to TFBQ adsorption}

The adsorption of organic molecules on metal surfaces often perturbs the electronic structure of the surface atoms, in particular if a (partially covalent) bond is formed. As a result, the surface metal atoms often rearrange. The extent of these rearrangements varies from relatively small (e.g., ca. $0.3 \AA$ for the adsorption of TCNQ on $\mathrm{Cu}^{9}$ ) to significantly more than $1.0 \AA$ (TCNE on $\mathrm{Cu}^{10}$ ). In several cases, even the formation of surface adatoms has been observed. ${ }^{11,12}$ For large molecules bearing carboxyl groups (i.e., PTCDA), however, the 
effects is expected to be generally small. ${ }^{13}$ In this work, we observe larger surface atom rearrangements for the bond-forming TFBQ molecule than for the inert TFBQ. However, even there the rearrangements are relatively unspectacular. The difference in the mean zposition of the extracted metal atoms to the z-position of the other atoms in the uppermost metal layer is $0.13 \AA$ on $\mathrm{Cu}(111)$ and $0.05 \AA ̊$ on $\mathrm{Ag}(111)$. 


\section{G. TFBQ-MODOS}

From the molecular orbital projected density of states (MODOS) for a homogeneous monolayer of TFBQ adsorbed on $\mathrm{Cu}(111)$ and $\mathrm{Ag}(111)$ shown in Figure S10 it is apparent that the LUMO-peak is located at the Fermi level for both cases.

(a) $\mathrm{Cu}(111)$

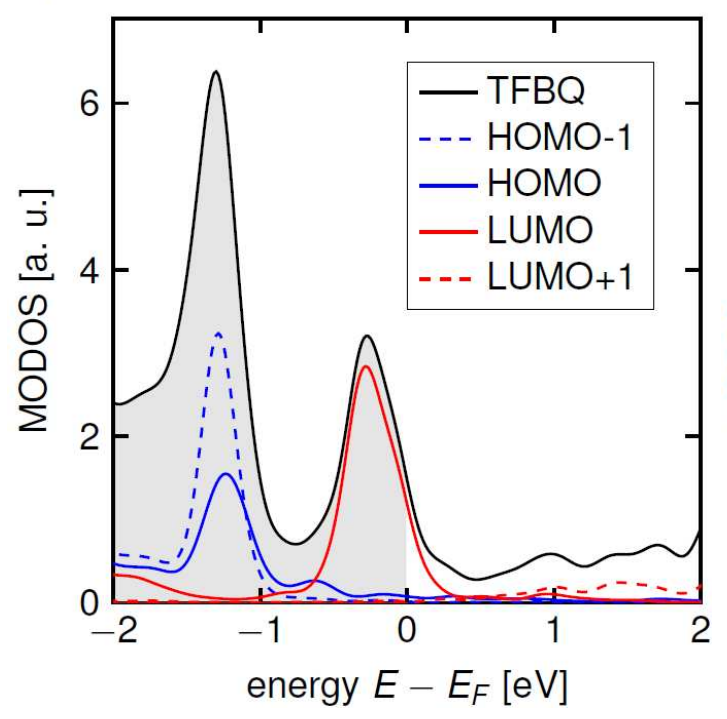

(b) $\mathrm{Ag}(111)$

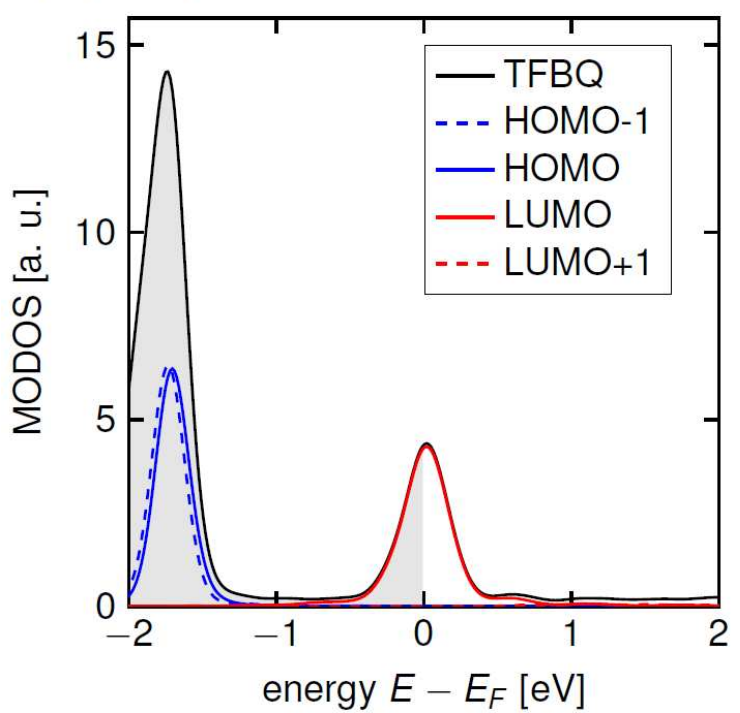

Figure S10. MODOS for a homogenous monolayer of TFBQ adsorbed on (a) Cu(111) and (b) $\operatorname{Ag}(111)$.

(1) Otero, R.; Écija, D.; Fernández, G.; Gallego, J. M.; Sánchez, L.; Martín, N.; Miranda, R. Nano Lett. 2007, 7 (9), 2602-2607.

(2) Stranick, S. J.; Parikh, A. N.; Tao, Y.-T.; Allara, D. L.; Weiss, P. S. J. Phys. Chem. 1994, 98 (31), 7636-7646.

(3) Stadtmüller, B.; Lüftner, D.; Willenbockel, M.; Reinisch, E. M.; Sueyoshi, T.; Koller, G.; Soubatch, S.; Ramsey, M. G.; Puschnig, P.; Tautz, F. S.; Kumpf, C. Nat. Commun. 2014, 5.

(4) Mulliken, R. S. J. Chem. Phys. 1955, 23 (10), 1833.

(5) Atalla, V.; Yoon, M.; Caruso, F.; Rinke, P.; Scheffler, M. Phys. Rev. B 2013, 88 (16).

(6) Avilov, I.; Geskin, V.; Cornil, J. Adv. Funct. Mater. 2009, 19 (4), 624-633.

(7) Hofmann, O. T.; Atalla, V.; Moll, N.; Rinke, P.; Scheffler, M. New J. Phys. 2013, 15 (12), 123028.

(8) Stein, S. E. In NIST Chemistry WebBook; NIST Standard Reference Database Number 69; Eds. P.J. Linstrom and W.G. Mallard: Gaithersburg MD.

(9) Tseng, T.-C.; Urban, C.; Wang, Y.; Otero, R.; Tait, S. L.; Alcamí, M.; Écija, D.; Trelka, M.; Gallego, J. M.; Lin, N.; et al. Charge-Transfer-Induced Structural Rearrangements at Both Sides of Organic/metal Interfaces. Nat. Chem. 2010, 2 (5), 374-379.

(10) Bedwani, S.; Wegner, D.; Crommie, M. F.; Rochefort, A. Strongly Reshaped OrganicMetal Interfaces: Tetracyanoethylene on $\mathrm{Cu}(100)$. Phys. Rev. Lett. 2008, 101 (21). 
(11) Faraggi, M. N.; Jiang, N.; Gonzalez-Lakunza, N.; Langner, A.; Stepanow, S.; Kern, K.; Arnau, A. Bonding and Charge Transfer in Metal-Organic Coordination Networks on Au(111) with Strong Acceptor Molecules. J. Phys. Chem. C 2012, 116 (46), 2455824565.

(12) Tait, S. L.; Langner, A.; Lin, N.; Stepanow, S.; Rajadurai, C.; Ruben, M.; Kern, K. OneDimensional Self-Assembled Molecular Chains on $\mathrm{Cu}(100)$ : Interplay between SurfaceAssisted Coordination Chemistry and Substrate Commensurability. J. Phys. Chem. C 2007, 111 (29), 10982-10987.

(13) Tautz, F. S. Structure and Bonding of Large Aromatic Molecules on Noble Metal Surfaces: The Example of PTCDA. Prog. Surf. Sci. 2007, 82 (9-12), 479-520. 